\title{
The numerical solution of partial differential-algebraic equations
}

\author{
Muhammed Yigider ${ }^{1 *}$ and Ercan Çelik ${ }^{2}$
}

${ }^{\text {*Correspondence: }}$

muhammed.yigider@erzurum.edu.tr

1 Department of Mathematics,

Faculty of Science, Erzurum

Technical University, Erzurum,

Turkey

Full list of author information is

available at the end of the article

\begin{abstract}
In this paper, a numerical solution of partial differential-algebraic equations (PDAEs) is considered by multivariate Padé approximations. We applied this method to an example. First, PDAE has been converted to power series by two-dimensional differential transformation, and then the numerical solution of the equation was put into a multivariate Padé series form. Thus, we obtained the numerical solution of PDAEs.
\end{abstract}

Keywords: partial differential-algebraic equation (PDAE); two-dimensional differential transformation; multivariate Padé approximation

\section{Introduction}

In this study, we consider linear partial differential-algebraic equations (PDAEs) of the form

$$
A u_{t}(t, x)+B u_{x x}(t, x)+C u(t, x)=f(t, x)
$$

where $t \in\left(0, t_{e}\right)$ and $x \in(-l, l) \subset R, A, B, C \in R^{n, x n}$ are constant matrices, $u, f:\left[0, t_{e}\right] \times$ $[-l, l] \rightarrow R^{n}$. We are interested in cases where at least one of the matrices, $A$ or $B$, is singular. The two special cases $A=0$ or $B=0$ lead to ordinary differential equations or DAEs which are not considered here. Therefore, in this paper we assume that none of the matrices $A$ or $B$ is the zero matrix [1-3]. Many important mathematical models can be expressed in terms of PDAEs. Such models arise in many areas of mathematics, engineering, the physical sciences and population growth. In recent years, much research has been focused on the numerical solution of PDAEs $[4,5]$. Some numerical methods have been developed using Runge-Kutta methods [6,7]. The purpose of this paper is to consider the numerical solution of PDAEs by using multivariate Padé approximations.

\section{Two-dimensional differential transformation}

The basic definition of the two-dimensional differential transform is given as follows [8-13]:

$$
W(k, h)=\frac{1}{k ! h !}\left[\frac{\partial^{k+h} w(x, y)}{\partial x^{k} \partial y^{h}}\right]_{0,0},
$$

where $w(x, y)$ is the original function and $W(k, h)$ is the transformed function. The transformation is called $T$-function and lower case and upper case letters represent the original

\section{空 Springer}

(c) 2013 Yigider and Çelik; licensee Springer. This is an Open Access article distributed under the terms of the Creative Commons Attribution License (http://creativecommons.org/licenses/by/2.0), which permits unrestricted use, distribution, and reproduction in any medium, provided the original work is properly cited. 
and transformed functions respectively. The differential inverse transform of $W(k, h)$ is defined as

$$
w(x, y)=\sum_{k=0}^{\infty} \sum_{h=0}^{\infty} W(k, h) x^{k} y^{h}
$$

and from Equations (2) and (3) can be concluded

$$
w(x, y)=\sum_{k=0}^{\infty} \sum_{h=0}^{\infty} \frac{1}{k ! h !}\left[\frac{\partial^{k+h} w(x, y)}{\partial x^{k} \partial y^{h}}\right]_{0,0} x^{k} y^{h} .
$$

\section{Multivariate Padé approximants}

Consider the bivariate function $f(x, y)$ with Taylor series development

$$
f(x, y)=\sum_{i, j=0}^{\infty} c_{i j} x^{i} y^{j}
$$

around the origin. We know that a solution of the univariate Padé approximation problem for

$$
f(x)=\sum_{i=0}^{\infty} c_{i} x^{i}
$$

is given by

$$
p(x)=\left|\begin{array}{cccc}
\sum_{i=0}^{m} c_{i} x^{i} & x \sum_{i=0}^{m-1} c_{i} x^{i} & \ldots & x^{n} \sum_{i=0}^{m-n} c_{i} x^{i} \\
c_{m+1} & c_{m} & \ldots & c_{m+1-n} \\
\vdots & \vdots & \ddots & \vdots \\
c_{m+n} & c_{m+n-1} & \ldots & c_{m}
\end{array}\right|
$$

and

$$
q(x)=\left|\begin{array}{cccc}
1 & x & \cdots & x^{n} \\
c_{m+1} & c_{m} & \cdots & c_{m+1-n} \\
\vdots & \vdots & \ddots & \vdots \\
c_{m+n} & c_{m+n-1} & \cdots & c_{m}
\end{array}\right|
$$

Let us now multiply the $j$ th row in $p(x)$ and $q(x)$ by $x^{j+m-1}(j=2, \ldots, n+1)$ and afterwards divide the $j$ th column in $p(x)$ and $q(x)$ by $x^{j-1}(j=2, \ldots, n+1)$. This results in a multiplication 
of numerator and denominator by $x^{m n}$. Having done so, we get

$$
\frac{p(x)}{q(x)}=\frac{\left|\begin{array}{cccc}
\sum_{i=0}^{m} c_{i} x^{i} & \sum_{i=0}^{m-1} c_{i} x^{i} & \cdots & \sum_{i=0}^{m-n} c_{i} x^{i} \\
c_{m+1} x^{m+1} & c_{m} x^{m} & \cdots & c_{m+1-n} x^{m+1-n} \\
\vdots & \vdots & \ddots & \vdots \\
c_{m+n} x^{m+n} & c_{m+n-1} x^{m+n-1} & \cdots & c_{m} x^{m}
\end{array}\right|}{\left|\begin{array}{cccc}
1 & 1 & \cdots & 1 \\
c_{m+1} x^{m+1} & c_{m} x^{m} & \cdots & c_{m+1-n} x^{m+1-n} \\
\vdots & \vdots & \ddots & \vdots \\
c_{m+n} x^{m+n} & c_{m+n-1} x^{m+n-1} & \cdots & c_{m} x^{m}
\end{array}\right|}
$$

if $\left(D=\operatorname{det} D_{m, n} \neq 0\right)$.

This quotient of determinants can also immediately be written down for a bivariate function $f(x, y)$. The sum $\sum_{i=0}^{k} c_{i} x^{i}$ will be replaced by the $k$ th partial sum of the Taylor series development of $f(x, y)$ and the expression $c_{k} x^{k}$ by an expression that contains all the terms of degree $k$ in $f(x, y)$. Here a bivariate term $c_{i j} x^{i} y^{j}$ is said to be of degree $i+j$.

If we define

$$
p(x, y)=\left|\begin{array}{cccc}
\sum_{i+j=0}^{m} c_{i j} x^{i} y^{j} & \sum_{i+j=0}^{m-1} c_{i j} x^{i} y^{j} & \ldots & \sum_{i+j=0}^{m-n} c_{i j} x^{i} y^{j} \\
\sum_{i+j=m+1} c_{i j} x^{i} y^{j} & \sum_{i+j=m} c_{i j} x^{i} y^{j} & \cdots & \sum_{i+j=m+1-n} c_{i j} x^{i} y^{j} \\
\vdots & \vdots & \ddots & \vdots \\
\sum_{i+j=m+n} c_{i j} x^{i} y^{j} & \sum_{i+j=m+n-1}^{m} c_{i j} x^{i} y^{j} & \ldots & \sum_{i+j=m}^{m} c_{i j} x^{i} y^{j}
\end{array}\right|
$$

and

$$
q(x, y)=\left|\begin{array}{cccc}
1 & 1 & \cdots & 1 \\
\sum_{i+j=m+1} c_{i j} x^{i} y^{j} & \sum_{i+j=m} c_{i j} x^{i} y^{j} & \cdots & \sum_{i+j=m+1-n} c_{i j} x^{i} y^{j} \\
\vdots & \vdots & \ddots & \vdots \\
\sum_{i+j=m+n} c_{i j} x^{i} y^{j} & \sum_{i+j=m+n-1}^{m} c_{i j} x^{i} y^{j} & \cdots & \sum_{i+j=m}^{m} c_{i j} x^{i} y^{j}
\end{array}\right|,
$$

then it is easy to see that $p(x, y)$ and $q(x, y)$ are of the form

$$
\begin{aligned}
& p(x, y)=\sum_{i+j=m n}^{m n+m} a_{i j} x^{i} y^{j}, \\
& q(x, y)=\sum_{i+j=m n}^{m n+n} b_{i j} x^{i} y^{j} .
\end{aligned}
$$

We know that $p(x, y)$ and $q(x, y)$ are called Padé equations $[3,14]$. So, the multivariate Padé approximant of order $(m, n)$ for $f(x, y)$ is defined as

$$
r_{m, n}(x, y)=\frac{p(x, y)}{q(x, y)} .
$$




\section{Numerical example}

The test problem considers the following PDAE [6]:

$$
\begin{aligned}
& \left(\begin{array}{ccc}
0 & 2 & 0 \\
1 & -1 & 0 \\
1 & -1 & 0
\end{array}\right) u_{t}+\left(\begin{array}{ccc}
-1 & 0 & 0 \\
0 & 0 & 0 \\
0 & 0 & -1
\end{array}\right) u_{x x}+\left(\begin{array}{ccc}
0 & 0 & 0 \\
0 & -1 & 0 \\
0 & 0 & 1
\end{array}\right) u=f, \\
& x \in[-0.5,0.5], t \in[0,1],
\end{aligned}
$$

where

$$
\begin{aligned}
& f_{1}=-x^{2} e^{-\frac{1}{2} t}-2 e^{-t} \\
& f_{2}=-x^{2} e^{-t}-\frac{1}{2} x^{2} e^{-\frac{1}{2} t} \\
& f_{3}=-x^{2} e^{-t}+\frac{1}{2} x^{2} e^{-\frac{1}{2} t}+\left(x^{2}-2\right) \sin t
\end{aligned}
$$

The exact solution is

$$
u(x, t)=\left(\begin{array}{c}
x^{2} e^{t} \\
x^{2} e^{-\frac{1}{2} t} \\
x^{2} \sin t
\end{array}\right)
$$

Equivalently, Equation (14) can be written as

$$
\left(\begin{array}{ccc}
0 & 2 & 0 \\
1 & -1 & 0 \\
1 & -1 & 0
\end{array}\right)\left(\begin{array}{l}
u_{1 t} \\
u_{2 t} \\
u_{3 t}
\end{array}\right)+\left(\begin{array}{ccc}
-1 & 0 & 0 \\
0 & 0 & 0 \\
0 & 0 & -1
\end{array}\right)\left(\begin{array}{l}
u_{1 x x} \\
u_{2 x x} \\
u_{3 x x}
\end{array}\right)+\left(\begin{array}{ccc}
0 & 0 & 0 \\
0 & -1 & 0 \\
0 & 0 & 1
\end{array}\right)\left(\begin{array}{l}
u_{1} \\
u_{2} \\
u_{3}
\end{array}\right)=\left(\begin{array}{l}
f_{1} \\
f_{2} \\
f_{3}
\end{array}\right)
$$

By using the basic definition of the two-dimensional differential transform and taking the transform of Equation (16), we can obtain that

$$
\begin{aligned}
& 2(k+1) U_{2}(k+1, h)-(h+1)(h+2) U_{1}(k, h+2)=F_{1}(k, h), \\
& (k+1) U_{1}(k+1, h)-(k+1) U_{2}(k+1, h)-U_{2}(k, h)=F_{2}(k, h), \\
& (k+1) U_{1}(k+1, h)-(k+1) U_{2}(k+1, h)-(h+1)(h+2) U_{3}(k, h+2)+U_{3}(k, h) \\
& \quad=F_{3}(k, h) .
\end{aligned}
$$

Consequently, by substituting the values of $u_{i}$, we have obtained

$$
\begin{aligned}
& u_{1}(x, t)=x^{2}-x^{2} t+\frac{1}{2} x^{2} t^{2}-\frac{1}{6} x^{2} t^{3}+\frac{1}{24} x^{2} t^{4}-\frac{1}{120} x^{2} t^{5}+\frac{1}{720} x^{2} t^{6} \\
& u_{2}(x, t)=x^{2}-\frac{1}{2} x^{2} t+\frac{1}{8} x^{2} t^{2}-\frac{1}{48} x^{2} t^{3}+\frac{1}{384} x^{2} t^{4}-\frac{1}{3840} x^{2} t^{5}-\frac{1}{46080} x^{2} t^{6} \\
& u_{3}(x, t)=x^{2} t-\frac{1}{6} x^{2} t^{3}+\frac{1}{120} x^{2} t^{5}
\end{aligned}
$$


Table 1 Comparison of the numerical solution of $u_{1}(x, t)$ with exact solutions $(t=0.01)$

\begin{tabular}{llll}
\hline $\boldsymbol{x}$ & $\boldsymbol{u}_{\mathbf{1}}(\boldsymbol{x}, \boldsymbol{t})$ & $\boldsymbol{r}_{\mathbf{4}, \mathbf{3}}(\boldsymbol{x}, \boldsymbol{t})$ & $\left|\boldsymbol{u}_{\mathbf{1}}(\boldsymbol{x}, \boldsymbol{t})-\boldsymbol{r}_{\mathbf{4}, \mathbf{3}}(\boldsymbol{x}, \boldsymbol{t})\right|$ \\
\hline-0.5 & 0.2475124584 & 0.2475124584 & 0 \\
-0.4 & 0.1584079734 & 0.1584079734 & 0 \\
-0.3 & 0.08910448503 & 0.08910448502 & $1.10^{-11}$ \\
-0.2 & 0.03960199335 & 0.03960199334 & $1.10^{-11}$ \\
-0.1 & 0.009900498337 & 0.009900498336 & $1.10^{-12}$ \\
0.1 & 0.009900498337 & 0.009900498336 & $1.10^{-12}$ \\
0.2 & 0.03960199335 & 0.03960199334 & $1.10^{-11}$ \\
0.3 & 0.08910448503 & 0.08910448502 & $1.10^{-11}$ \\
0.4 & 0.1584079734 & 0.1584079734 & 0 \\
0.5 & 0.2475124584 & 0.2475124584 & 0 \\
\hline
\end{tabular}

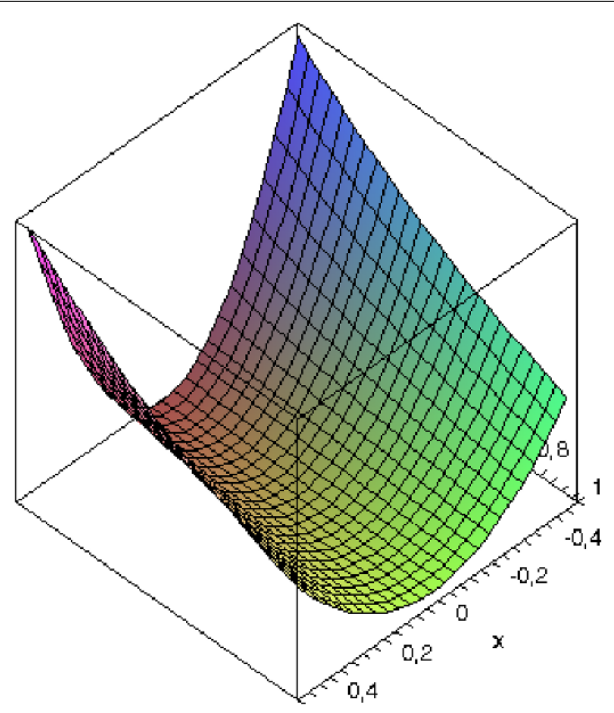

Figure 1 Values of $u_{1}(x, t)$.

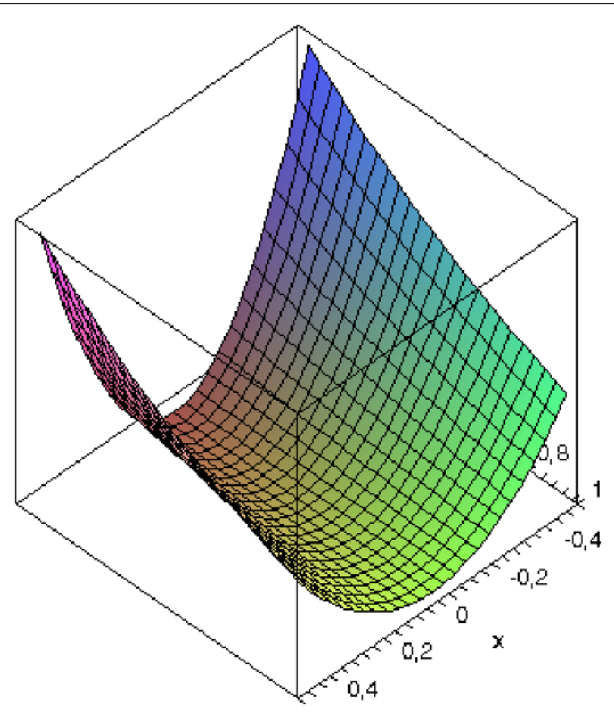

Figure 2 Values of $r_{4,3}(x, t)$ Padé approximant. 
Table 2 Comparison of the numerical solution of $u_{2}(x, t)$ with exact solutions $(t=0.01)$

\begin{tabular}{llll}
\hline $\boldsymbol{x}$ & $\mathbf{u}_{\mathbf{2}}(\boldsymbol{x}, \boldsymbol{t})$ & $\boldsymbol{r}_{\mathbf{4}, \mathbf{3}}(\boldsymbol{x}, \boldsymbol{t})$ & $\left|\mathbf{u}_{\mathbf{2}}(\boldsymbol{x}, \boldsymbol{t})-\boldsymbol{r}_{\mathbf{4}, \mathbf{3}}(\boldsymbol{x}, \boldsymbol{t})\right|$ \\
\hline-0.5 & 0.2487531198 & 0.2487531198 & 0 \\
-0.4 & 0.1592019967 & 0.1592019967 & 0 \\
-0.3 & 0.08955112313 & 0.08955112314 & $1.10^{-11}$ \\
-0.2 & 0.03980049917 & 0.03980049917 & 0 \\
-0.1 & 0.009950124792 & 0.009950124793 & $1.10^{-12}$ \\
0.1 & 0.009950124792 & 0.009950124793 & $1.10^{-12}$ \\
0.2 & 0.03980049917 & 0.03980049917 & 0 \\
0.3 & 0.08955112313 & 0.08955112314 & $1.10^{-12}$ \\
0.4 & 0.1592019967 & 0.1592019967 & 0 \\
0.5 & 0.2487531198 & 0.2487531198 & 0 \\
\hline
\end{tabular}

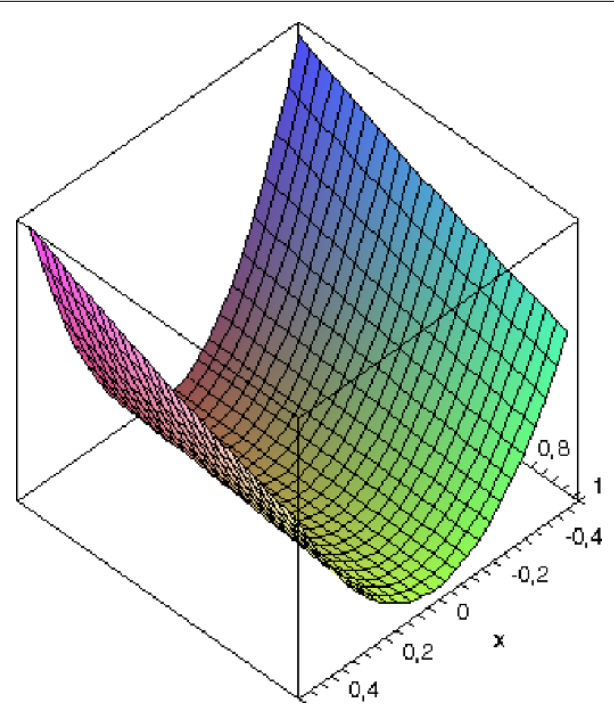

Figure 3 Values of $u_{2}(x, t)$.

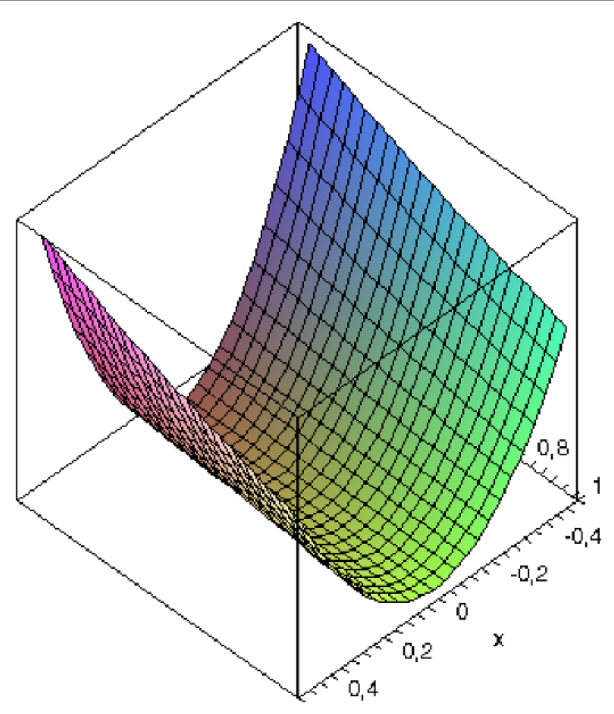

Figure 4 Values of $r_{4,3}(x, t)$ Padé approximant. 
Table 3 Comparison of the numerical solution of $u_{3}(x, t)$ with exact solutions $(t=0.01)$

\begin{tabular}{rlll}
\hline $\boldsymbol{x}$ & $\boldsymbol{u}_{\mathbf{3}}(\boldsymbol{x}, \boldsymbol{t})$ & $\boldsymbol{r}_{\mathbf{4}, \mathbf{3}}(\boldsymbol{x}, \boldsymbol{t})$ & $\left|\mathbf{u}_{\mathbf{3}}(\boldsymbol{x}, \boldsymbol{t})-\boldsymbol{r}_{\mathbf{4}, \mathbf{3}}(\boldsymbol{x}, \boldsymbol{t})\right|$ \\
\hline-0.5 & 0.002499958334 & 0.002499958333 & $1.10^{-12}$ \\
-0.4 & 0.001599973333 & 0.001599973333 & 0 \\
-0.3 & 0.0008999850001 & 0.0008999850000 & $1.10^{-13}$ \\
-0.2 & 0.0003999933334 & 0.0003999933333 & $1.10^{-13}$ \\
-0.1 & 0.00009999833334 & 0.00009999833333 & $1.10^{-14}$ \\
0.1 & 0.00009999833334 & 0.00009999833333 & $1.10^{-14}$ \\
0.2 & 0.0003999933334 & 0.0003999933333 & $1.10^{-13}$ \\
0.3 & 0.0008999850001 & 0.0008999850000 & $1.10^{-13}$ \\
0.4 & 0.001599973333 & 0.001599973333 & 0 \\
0.5 & 0.002499958334 & 0.002499958333 & $1.10^{-12}$ \\
\hline
\end{tabular}

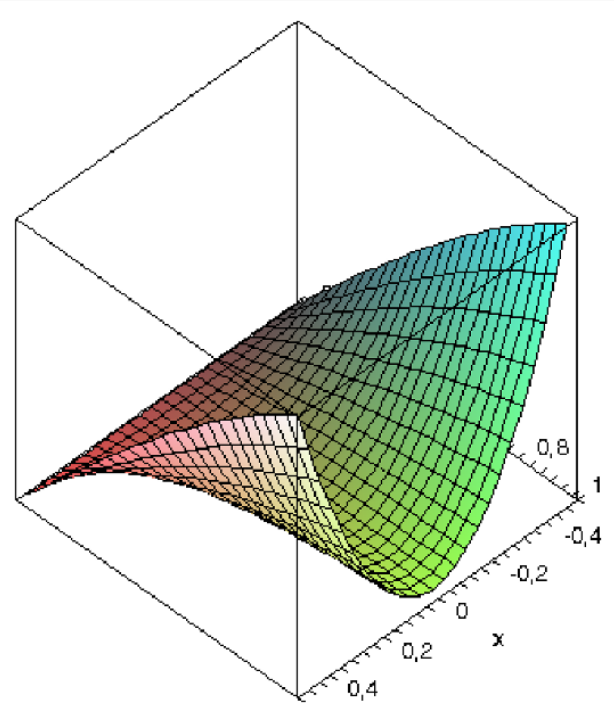

Figure 5 Values of $u_{3}(x, t)$.

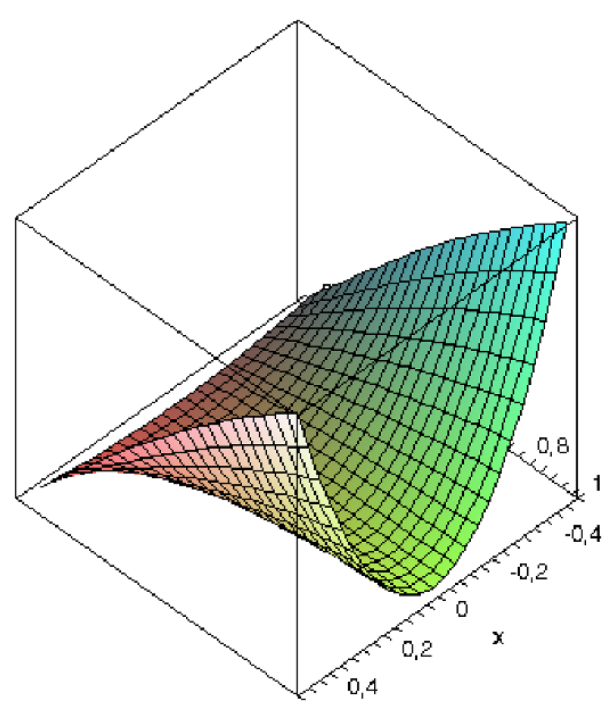

Figure 6 Values of $r_{4,3}(x, t)$ Padé approximant. 
The power series $u_{1}(x, t), u_{2}(x, t)$ and $u_{3}(x, t)$ can be transformed into multivariate Padé approximation

$$
\begin{aligned}
& m=4, \quad n=3, \\
& p_{1}(x, t)=\left|\begin{array}{cccc}
x^{2}-x^{2} t+\frac{1}{2} x^{2} t^{2} & x^{2}-x^{2} t & x^{2} & 0 \\
-\frac{1}{6} x^{2} t^{3} & \frac{1}{2} x^{2} t^{2} & -x^{2} t & x^{2} \\
\frac{1}{24} x^{2} t^{4} & -\frac{1}{6} x^{2} t^{3} & \frac{1}{2} x^{2} t^{2} & -x^{2} t \\
\frac{1}{120} x^{2} t^{5} & \frac{1}{24} x^{2} t^{4} & -\frac{1}{6} x^{2} t^{3} & \frac{1}{2} x^{2} t^{2}
\end{array}\right| \\
& =\frac{1}{144} x^{8} t^{6}-\frac{1}{360} x^{8} t^{7}+\frac{1}{2880} x^{8} t^{8} \\
& =0.006944444444 x^{8} t^{6}+0.002777777778 x^{8} t^{7}+0.0003472222222 x^{8} t^{8} \text {, } \\
& q_{1}(x, t)=\left|\begin{array}{cccc}
1 & 1 & 1 & 1 \\
-\frac{1}{6} x^{2} t^{3} & \frac{1}{2} x^{2} t^{2} & -x^{2} t & x^{2} \\
\frac{1}{24} x^{2} t^{4} & -\frac{1}{6} x^{2} t^{3} & \frac{1}{2} x^{2} t^{2} & -x^{2} t \\
\frac{1}{120} x^{2} t^{5} & \frac{1}{24} x^{2} t^{4} & -\frac{1}{6} x^{2} t^{3} & \frac{1}{2} x^{2} t^{2}
\end{array}\right| \\
& =\frac{1}{144} x^{6} t^{6}+\frac{1}{240} x^{6} t^{7}+\frac{1}{960} x^{6} t^{8}+\frac{1}{8640} x^{8} t^{8} \\
& =0.006944444444 x^{6} t^{6}+0.004166666667 x^{6} t^{7}+0.001041666667 x^{6} t^{8} \\
& +0.0001157407407 x^{8} t^{8}, \\
& r_{1}(x, t)=\left(\frac{1}{144} x^{8} t^{6}-\frac{1}{360} x^{8} t^{7}+\frac{1}{2880} x^{8} t^{8}\right) \\
& /\left(\frac{1}{144} x^{6} t^{6}+\frac{1}{240} x^{6} t^{7}+\frac{1}{8640} x^{8} t^{8}+\frac{1}{960}+x^{6} t^{8}\right) \\
& =\left(0.006944444444 x^{8} t^{6}+0.002777777778 x^{8} t^{7}+0.0003472222222 x^{8} t^{8}\right) \\
& /\left(0.006944444444 x^{6} t^{6}+0.004166666667 x^{6} t^{7}+0.0001157407407 x^{8} t^{8}\right. \\
& \left.+0.001041666667 x^{6} t^{8}\right), \\
& p_{2}(x, t)=\left|\begin{array}{cccc}
x^{2}-\frac{1}{2} x^{2} t+\frac{1}{8} x^{2} t^{2} & x^{2}-\frac{1}{2} x^{2} t & x^{2} & 0 \\
-\frac{1}{48} x^{2} t^{3} & \frac{1}{8} x^{2} t^{2} & -\frac{1}{2} x^{2} t & x^{2} \\
\frac{1}{384} x^{2} t^{4} & -\frac{1}{48} x^{2} t^{3} & \frac{1}{8} x^{2} t^{2} & -\frac{1}{2} x^{2} t \\
\frac{1}{3840} x^{2} t^{5} & \frac{1}{384} x^{2} t^{4} & -\frac{1}{48} x^{2} t^{3} & \frac{1}{8} x^{2} t^{2}
\end{array}\right| \\
& =\frac{1}{9216} x^{8} t^{6}-\frac{1}{46080} x^{8} t^{7}+\frac{1}{737280} x^{8} t^{8} \\
& =0.0001085069444 x^{8} t^{6}-0.00002170138889 x^{8} t^{7} \\
& +0.000001356336806 x^{8} t^{8}, \\
& q_{2}(x, t)=\left|\begin{array}{cccc}
1 & 1 & 1 & 1 \\
-\frac{1}{48} x^{2} t^{3} & \frac{1}{8} x^{2} t^{2} & -\frac{1}{2} x^{2} t & x^{2} \\
\frac{1}{384} x^{2} t^{4} & -\frac{1}{48} x^{2} t^{3} & \frac{1}{8} x^{2} t^{2} & -\frac{1}{2} x^{2} t \\
\frac{1}{3840} x^{2} t^{5} & \frac{1}{384} x^{2} t^{4} & -\frac{1}{48} x^{2} t^{3} & \frac{1}{8} x^{2} t^{2}
\end{array}\right| \\
& =\frac{1}{9216} x^{6} t^{6}+\frac{1}{30720} x^{6} t^{7}+\frac{1}{245760} x^{6} t^{8}+\frac{1}{4423680} x^{6} t^{9}
\end{aligned}
$$




$$
\begin{aligned}
& =0.0001085069444 x^{6} t^{6}+0.00003255208333 x^{6} t^{7} \\
& +0.000004069010417 x^{6} t^{8}+0.0000002260561343 x^{6} t^{9}, \\
& r_{2}(x, t)=\left(\frac{1}{9216} x^{8} t^{6}-\frac{1}{46080} x^{8} t^{7}+\frac{1}{737280} x^{8} t^{8}\right) \\
& /\left(\frac{1}{9216} x^{6} t^{6}+\frac{1}{30720} x^{6} t^{7}+\frac{1}{245760} x^{6} t^{8}+\frac{1}{4423680} x^{6} t^{9}\right) \\
& =\left(0.0001085069444 x^{8} t^{6}-0.00002170138889 x^{8} t^{7}\right. \\
& \left.+0.000001356336806 x^{8} t^{8}\right) \\
& /\left(0.0001085069444 x^{6} t^{6}+0.00003255208333 x^{6} t^{7}\right. \\
& \left.+0.000004069010417 x^{6} t^{8}+0.0000002260561343 x^{6} t^{9}\right) \text {, } \\
& p_{3}(x, t)=\left|\begin{array}{cccc}
x^{2} t & x^{2} t & 0 & 0 \\
-\frac{1}{6} x^{2} t^{3} & 0 & x^{2} t & 0 \\
0 & -\frac{1}{6} x^{2} t^{3} & 0 & x^{2} t \\
\frac{1}{120} x^{2} t^{5} & 0 & -\frac{1}{6} x^{2} t^{3} & 0
\end{array}\right|=\frac{7}{360} x^{8} t^{8} \\
& =0.01944444444 x^{8} t^{8} \text {, } \\
& q_{3}(x, t)=\left|\begin{array}{cccc}
1 & 1 & 1 & 1 \\
-\frac{1}{6} x^{2} t^{3} & 0 & x^{2} t & 0 \\
0 & -\frac{1}{6} x^{2} t^{3} & 0 & x^{2} t \\
\frac{1}{120} x^{2} t^{5} & 0 & -\frac{1}{6} x^{2} t^{3} & 0
\end{array}\right|=\left(\frac{7}{360} x^{6} t^{7}+\frac{7}{2160} x^{6} t^{9}\right) \\
& =0.01944444444 x^{6} t^{7}+0.003240740741 x^{6} t^{9} \text {, } \\
& r_{3}(x, t)=\left(\frac{7}{360} x^{8} t^{8}\right) /\left(\frac{7}{360} x^{6} t^{7}+\frac{7}{2160} x^{6} t^{9}\right) \\
& =\left(0.01944444444 x^{8} t^{8}\right) /\left(0.01944444444 x^{6} t^{7}+0.003240740741 x^{6} t^{9}\right) .
\end{aligned}
$$

\section{Conclusions}

The method for solving partial differential-algebraic equations (PDAEs) has been proposed. The results of the example showed from Tables 1-3 and Figures 1-6 that exactly the same solutions have been obtained with multivariate Padé approximation. On the other hand, the results are quite reliable. Therefore, this method can be applied to many complicated PDAEs.

Competing interests

The authors declare that they have no competing interests.

\section{Authors' contributions}

All authors carried out the proof and conceived of the study. All authors read and approved the final manuscript.

\section{Author details}

${ }^{1}$ Department of Mathematics, Faculty of Science, Erzurum Technical University, Erzurum, Turkey. ${ }^{2}$ Department of Mathematics, Faculty of Science, Atatürk University, Erzurum, Turkey.

\section{Acknowledgements}

The authors thank the referees for valuable comments and suggestions which improved the presentation of this manuscript. This study was supported by The Scientific Research Projects of Atatürk University. 


\section{References}

1. Lucht, W, Strehmel, K, Liebenow, CE: Linear partial differential-algebraic equations, part I. Reports of the Institute of Numerical Mathematics, Report No. 17 (1997)

2. Lucht, W, Strehmel, K, Liebenow, CE: Linear partial differential-algebraic equations, part II. Reports of the Institute of Numerical Mathematics, Report No. 18 (1997)

3. Cuyt, A, Wuytack, L: Nonlinear Methods in Numerical Analysis. North-Holland, Amsterdam (1987)

4. Turut, V, Guzel, N: Comparing numerical methods for solving time-fractional reaction-diffusion equations. ISRN Math. Anal. (2012). doi:10.5402/2012/737206

5. Kurulay, M, Bayram, M: Approximate analytical solution for the fractional modified KdV by differential transform method. Commun. Nonlinear Sci. Numer. Simul. 15, 1777-1782 (2010)

6. Strehmel, K, Debrabant, K: Convergence of Runge-Kutta methods applied to linear partial differential-algebraic equations. Appl. Numer. Math. 53, 213-229 (2005)

7. Turut, V, Guzel, N: On solving partial differential equations of fractional order by using the variational iteration method and multivariate Padé approximation, EJPAM (2010, accepted)

8. Yiğider, M, Çelik, E: The numerical solution of partial differential-algebraic equations (PDAEs) by multivariate Padé approximation. Eur. J. Pure Appl. Math. 4, 67-75 (2011)

9. Zhou, JK: Differential Transform and Its Applications for Electrical Circuits. Huarjung University Press, Wuhan (1986)

10. Ayaz, F: On the two-dimensional differential transform method. Appl. Math. Comput. 143, 361-374 (2003)

11. Ayaz, F: Solutions of the system of differential equations by differential transform method. Appl. Math. Comput. 147, 547-567 (2004)

12. Bildik, N, Konuralp, A: Two-dimensional differential transform method, Adomian's decomposition method and variational iteration method for partial differential equations. Int. J. Comput. Math. 83(12), 973-987 (2006)

13. Adomian, G: Convergent series solution of nonlinear equations. J. Comput. Appl. Math. 11, 225-230 (1984)

14. Celik, E, Karaduman, E, Bayram, M: Numerical solutions of chemical differential-algebraic equations. Appl. Math. Comput. 139(2-3), 259-264 (2003)

doi:10.1186/1687-1847-2013-8

Cite this article as: Yigider and Çelik: The numerical solution of partial differential-algebraic equations. Advances in Difference Equations 2013 2013:8.

\section{Submit your manuscript to a SpringerOpen ${ }^{\ominus}$ journal and benefit from:}

- Convenient online submission

- Rigorous peer review

- Immediate publication on acceptance

- Open access: articles freely available online

- High visibility within the field

- Retaining the copyright to your article 